\title{
Abundance patterns of the light neutron-capture elements in very and extremely metal-poor stars ${ }^{\star}$
}

\author{
F. Spite ${ }^{1}$, M. Spite ${ }^{1}$, B. Barbuy ${ }^{2}$, P. Bonifacio ${ }^{1}$, E. Caffau ${ }^{1}$, and P. François ${ }^{1,3}$ \\ ${ }^{1}$ GEPI, Observatoire de Paris, PSL Research University, CNRS, Place Jules Janssen, 92190 Meudon, France \\ e-mail: monique.spite@obspm.fr \\ 2 Universidade de São Paulo, IAG, Rua do Matão 1226, Cidade Universitária, 05508-900, São Paulo, Brazil \\ ${ }^{3}$ Université de Picardie Jules Verne, 33 rue St-Leu, 80080 Amiens, France
}

Received 12 October 2017 / Accepted 23 December 2017

\begin{abstract}
Aims. The abundance patterns of the neutron-capture elements in metal-poor stars provide a unique record of the nucleosynthesis products of the earlier massive primitive objects.

Methods. We measured new abundances of so-called light neutron-capture of first peak elements using local thermodynamic equilibrium (LTE) 1D analysis; this analysis resulted in a sample of 11 very metal-poor stars, from $[\mathrm{Fe} / \mathrm{H}]=-2.5$ to $[\mathrm{Fe} / \mathrm{H}]=-3.4$, and one carbon-rich star, CS 22949-037 with $[\mathrm{Fe} / \mathrm{H}]=-4.0$. The abundances were compared to those observed in two classical metal-poor stars: the typical r-rich star CS 31082-001 $([\mathrm{Eu} / \mathrm{Fe}]>+1.0)$ and the r-poor star HD $122563([\mathrm{Eu} / \mathrm{Fe}]<0.0)$, which are known to present a strong enrichment of the first peak neutron-capture elements relative to the second peak.

Results. Within the first peak, the abundances are well correlated in analogy to the well-known correlation inside the abundances of the second-peak elements. In contrast, there is no correlation between any first peak element with any second peak element. We show that the scatter of the ratio of the first peak abundance over second peak abundance increases when the mean abundance of the second peak elements decreases from r-rich to r-poor stars.

We found two new r-poor stars that are very similar to HD 122563. A third r-poor star, CS 22897-008, is even more extreme; this star shows the most extreme example of first peak elements enrichment to date. On the contrary, another r-poor star (BD-18 5550) has a pattern of first peak elements that is similar to the typical r-rich stars CS 31082-001, however this star has some Mo enrichment. Conclusions. The distribution of the neutron-capture elements in our very metal-poor stars can be understood as the combination of at least two mechanisms: one that enriches the forming stars cloud homogeneously through the main r-process and leads to an element pattern similar to the r-rich stars, such as CS 31082-001; and another that forms mainly lighter, first peak elements.
\end{abstract}

Key words. stars: abundances - stars: population II - galaxy: halo

\section{Introduction}

The neutron-capture elements are mainly synthesised, as recorded by the distribution of their isotopes in the Sun, by two classical processes: either the slow $(s)$ or rapid $(r)$ process. Schematically, the heavy elements of the s-process could be built by slow neutron production of asymptotic giant branch stars (AGB) and the r-process elements could be built by a flux of high neutron density.

The chemical composition of the old metal-poor stars reflects the production of elements in the first phases of galactic evolution. The matter that formed these very old stars had been only enriched by still older short-lived massive objects. However the exact nature of the sites of production of the observed neutroncapture elements in these stars is still actively debated (Cowan et al. 2011). The focus of neutron-capture abundance studies in the old metal-poor stars is an attempt to constrain the sites of the nucleosynthesis of these elements.

The r-process elements are produced by a rapid addition of neutrons to the atomic nuclei. This is the case in

\footnotetext{
* Based on observations collected at the European Organisation for Astronomical Research in the Southern Hemisphere under ESO programme 165.N-0276(A), (PI R.Cayrel).
}

rather rare but productive processes such as the mergers of neutron stars (Kasen et al. 2017; Vangioni et al. 2016; Goriely et al. 2015; Wanajo et al. 2014; Freiburghaus et al. 1999) and of course in several kinds of explosive burning phases of supernovae (see e.g. Wanajo et al. 2011; Winteler et al. 2012; Aoki et al. 2017; Nishimura et al. 2017; Banerjee et al. 2017).

The s-process elements can be produced by the slow irradiations of abundant seed nuclei (e.g. iron nuclei) by the neutron flux delivered in either of two cases:

(i) By pulsating stars in a late evolutionary phase, i.e. AGB and super AGB stars (SAGB; Käppeler et al. 2011; Bisterzo et al. 2017). The initial mass of these SAGB stars has to be lower than $9 M_{\odot}$ to reach the AGB phase, but higher than about $6 M_{\odot}$ to have a lifetime short enough to be able to enrich the matter before the formation of the very old low mass stars observed today.

(ii) In fast rotating massive stars (FRMS); see e.g. Meynet et al. (2006), Hirschi et al. (2007).

The results of the r- or s-processes are very different, and not only by the resulting isotopic composition. For example, the neutrons slowly absorbed by seed nuclei in the FRMS process produce a characteristic pattern with dominating lower mass elements in the first peak (Frischknecht et al. 2016); an 
example is given by Yong et al. (2017), who have analysed a star in the globular cluster $\Omega$ Cen.

In the old metal-poor Galactic stars, at a given metallicity, the scatter of the abundance of the neutron capture elements relative to iron $[\mathrm{X} / \mathrm{Fe}]^{1}$, is huge (compared to the low scatter of other element ratios such as $[\mathrm{Ca} / \mathrm{Fe}])$, as noted for example by François et al. (2007); Spite \& Spite (2014). Some stars are very rich in neutron-capture elements; these are called r-rich stars if $[\mathrm{Eu} / \mathrm{Fe}]>0.3$, but others, at the same metallicity, are r-poor with $[\mathrm{Eu} / \mathrm{Fe}] \ll 0.0$. It has been shown that the pattern of the element abundances in the r-rich stars is characteristic of the classical $r$ process, (see for example Cowan et al. 2011). But the pattern of the elements of the second peak $(56 \leq \mathrm{Z} \leq 70)$, is rather similar in r-rich and r-poor stars (see also Cowan et al. 2011).

On the contrary, the abundance pattern of the light neutroncapture elements $(32<Z<56$, or first peak elements) is very different in the r-rich and the r-poor stars. Compared to the classical r-rich metal-poor stars (for example CS 31082-001) (Hill et al. 2002; Siqueira Mello et al. 2013) or CS 22892-052 (Sneden et al. 2003), some very metal-poor (VMP) stars such as HD 122563 (Honda et al. 2006, 2007; Montes et al. 2007) present a strong excess of light neutron-capture elements (see also: François et al. 2007; Peterson 2011, 2013).

The behaviour of the second peak elements and light neutron-capture elements $\mathrm{Sr}, \mathrm{Y}, \mathrm{Zr}$ (first peak elements with $\mathrm{Z}=38,39$, and 40) were presented for a homogeneous sample of old, very, and extremely metal-poor (EMP) stars by François et al. (2007). These stars were studied in the context of the ESO Large Programme "First Stars" (hereafter LP First Stars); see Cayrel et al. (2004) and Bonifacio et al. (2009). The aim of the present paper is an attempt to determine in the same stars the behaviour of the intermediate first peak elements from Mo to $\mathrm{Ag}(\mathrm{Z}=42$ to 47$)$, when possible.

\section{Observational data}

The spectral lines of the light neutron-capture elements measured in this work are all located in the near UV between 338 and $390 \mathrm{~nm}$. The spectra were obtained with the Very Large Telescope (VLT) and the spectrograph UVES (Dekker et al. 2000) as part of the LP First Stars. The resolving power $R$ was close to 47000 and the spectra were reduced using the UVES context (Ballester et al. 2000); more information can be found in Cayrel et al. (2004).

Generally, in VMP and EMP stars, only the abundances of the first peak elements $\mathrm{Sr}, \mathrm{Y}$, and $\mathrm{Zr}$ appear in the literature because the rather strong spectral lines of these elements are observable in the visible domain; there are few measurements of the abundance of the heavier first peak elements $\mathrm{Mo}, \mathrm{Ru}, \mathrm{Pd}$, and $\mathrm{Ag}$ because the absorption lines of these elements are located in the less accessible near UV domain (see Hansen \& Primas 2011; Hansen et al. 2012, 2014a; Aoki et al. 2017). In the 12 giant stars we studied, it was possible to measure the abundance of these elements for the first time because the spectrograph UVES is very efficient in the near UV and the $\mathrm{S} / \mathrm{N}$ of the spectra, even in this region, was rather good. In Table 1 we give the $\mathrm{S} / \mathrm{N}$ of the spectra measured at $385 \mathrm{~nm}$. Because of line crowding, it is not possible to measure the $\mathrm{S} / \mathrm{N}$ at lower wavelengths with precision, but from the ESO exposure time calculator of UVES, for

\footnotetext{
1 We adopt the classical notation that, for each element $\mathrm{X}, \mathrm{A}(\mathrm{X})=$ $\log \left(\mathrm{N}_{\mathrm{X}} / \mathrm{N}_{\mathrm{H}}\right)+12 ;[\mathrm{X} / \mathrm{H}]=\log \left(\mathrm{N}_{\mathrm{X}} / \mathrm{N}_{\mathrm{H}}\right)_{\text {star }}-\log \left(\mathrm{N}_{\mathrm{X}} / \mathrm{N}_{\mathrm{H}}\right)_{\text {Sun }}$, and $[\mathrm{X} / \mathrm{Fe}]=[\mathrm{X} / \mathrm{H}]-[\mathrm{Fe} / \mathrm{H}]$.
}

Table 1. For each star in column 2 the $\mathrm{S} / \mathrm{N}$ of the mean spectrum measured at $385 \mathrm{~nm}$ and in columns 3 to 6 the parameters of the adopted models are listed.

\begin{tabular}{lccccc}
\hline \hline Object & $\mathrm{S} / \mathrm{N}(385 \mathrm{~nm})$ & $T_{\text {eff }}$ & $\log g$ & $\mathrm{v}_{\mathrm{t}}$ & {$[\mathrm{M} / \mathrm{H}]$} \\
\hline HD 2796 & 280 & 4950 & 1.5 & 2.1 & -2.5 \\
HD 186478 & 190 & 4700 & 1.3 & 2.0 & -2.6 \\
BD-18 5550 & 210 & 4750 & 1.4 & 1.8 & -3.0 \\
BS 17569-049 & 125 & 4700 & 1.2 & 1.9 & -2.9 \\
CS 22873-055 & 135 & 4550 & 0.7 & 2.2 & -3.0 \\
CS 22873-166 & 130 & 4550 & 0.9 & 2.1 & -3.0 \\
CS 22896-154 & 125 & 5250 & 2.7 & 1.2 & -2.7 \\
CS 22897-008 & 155 & 4900 & 1.7 & 2.0 & -3.4 \\
CS 22949-037 & 135 & 4900 & 1.5 & 1.8 & -4.0 \\
CS 22953-003 & 150 & 5100 & 2.3 & 1.7 & -2.8 \\
CS 22966-057 & 100 & 5300 & 2.2 & 1.4 & -2.6 \\
CS 29518-051 & 190 & 5200 & 2.6 & 1.4 & -2.7 \\
\hline
\end{tabular}

the kind of stars here studied, the $\mathrm{S} / \mathrm{N}$ is about four times less at $340 \mathrm{~nm}$.

\section{Abundance analysis}

A classical analysis of the spectra was carried out using OSMARCS model atmospheres (e.g. Gustafsson et al. 1975, 2003, 2008). The synthetic profiles were computed using the local thermodynamic equilibrium (LTE) spectral line analysis code turbospectrum (Alvarez \& Plez 1998; Plez 2012). The atmospheric parameters (Table 1) were taken from Cayrel et al. (2004). Examples of fits of neutron capture element profiles are shown in Fig. 1.

In Tables 2 and 3 we list the abundances of the neutroncapture elements of the first peak with the abundances of $\mathrm{Ba}$ and Eu taken from François et al. (2007). One of these stars, CS 22949-037 (Depagne et al. 2002), is a peculiar star; it is EMP, but $\mathrm{C}$-rich and r-poor. The abundances of $\mathrm{Eu}$ and $\mathrm{Sm}$ given in Depagne et al. (2002) are only upper limits, and Ba is the only second-peak element whose abundance could be measured in this star.

The absorption line of $\mathrm{Ag}$ at $338.2 \mathrm{~nm}$ is severely blended in the spectrum by a line of the $\mathrm{NH}$ molecule at practically the same wavelength. This $\mathrm{NH}$ line is relatively strong in the mixed (evolved) giants, where a mixing occurs between the atmosphere and the H-burning layer where $\mathrm{C}$ is converted into $\mathrm{N}$ by the $\mathrm{CNO}$ cycle. In order to have the best representation of the $\mathrm{NH}$ band in this region, it is necessary to use the abundance of nitrogen deduced from the $\mathrm{NH}$ band and not the $\mathrm{CN}$ band, since the abundance of $\mathrm{N}$ (deduced from the $\mathrm{CN}$ molecule) is, in these stars, systematically lower by about 0.4 dex than the abundance deduced from the NH molecule (Spite et al. 2005). In any case, the abundance of $\mathrm{Ag}$ is very uncertain in such N-rich stars where the contribution of $\mathrm{Ag}$ to the absorption feature is small (Fig. 1, bottom panel) .

In Table 3 we give the abundances of these elements relative to iron. The solar abundances adopted for these computations are from Lodders et al. (2009). In François et al. (2007) the solar abundances of Grevesse \& Sauval (2000) were adopted; the small difference in the adopted solar abundances mainly explains the small differences in the $[\mathrm{X} / \mathrm{Fe}]$ values between the tables of François et al. (2007) and the values given in Table 3. In some rare cases, additional measurements were carried out 

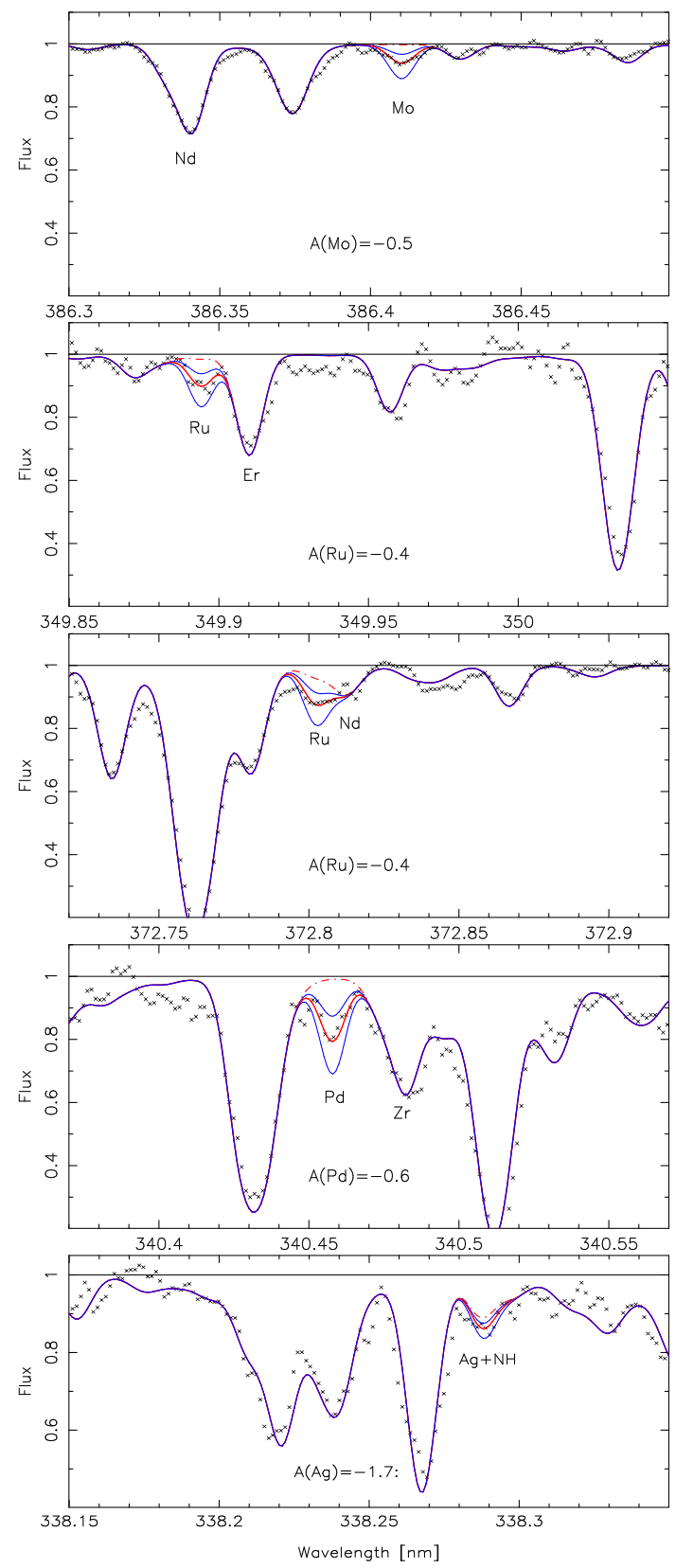

Fig. 1. Observed profile of the lines of Mo, Ru, $\mathrm{Pd}$, and $\mathrm{Ag}$ in BS 17569 049. Crosses indicate the observations. The synthetic profiles were computed with three options: an absence of the neutron-capture element (red dash-dotted line), the adopted abundance indicated in the figure (red thick line), and changing this adopted abundance by \pm 0.3 dex (blue thin lines). BS 17569-049 is a mixed star (therefore with a high abundance of nitrogen) and, as a consequence, the abundance of Ag deduced from the blended feature at $338.28 \mathrm{~nm}$ is very uncertain.

because new spectra became available, enabling an increase in the S/N. At the end of Tables 2 and 3, we give, as a comparison, the abundances of the heavy elements in the two extreme stars CS 31082-001 (Siqueira Mello et al. 2013) and in HD 122563 (Honda et al. 2007). These stars have almost the same metallicity $(-2.9$ and -2.8 , respectively), but the $[\mathrm{Ba} / \mathrm{Fe}]$ and $[\mathrm{Sr} / \mathrm{Ba}]$ ratios are very different.

\subsection{Comparison of our measurements with previous results}

No stars in our sample are in common with the sample of Aoki et al. (2017) and only one star is in common with those of Hansen et al. (2012, 2014a): HD 186478. With a metallicity $[\mathrm{Fe} / \mathrm{H}]=-2.59$ this star is one of the less metal-poor stars of our sample. Our abundances for HD 186478 agree with the values given by Hansen et al. (2012, 2014a) within the errors, except Ag. HD 186478 is a cool giant star with an important mixing between the $\mathrm{H}$ burning layer and the surface, and the abundance of nitrogen deduced from the $N H$ band reaches $[\mathrm{N} / \mathrm{Fe}] \approx+1.0$ in this star. With this high value of the nitrogen abundance, the $\mathrm{NH}$ molecule is able to explain fully the feature at $338.3 \mathrm{~nm}$ (see Fig. 1, bottom panel for another mixed star). Generally speaking we decided not to take into account the abundance of $\mathrm{Ag}$ in the mixed giants.

\subsection{Error budget}

The error in $[\mathrm{X} / \mathrm{Fe}]$ linked to our choice of the model parameters was computed as a quadratic sum of the uncertainties on $T_{\text {eff }}(100 \mathrm{~K}), \log g(0.2 \mathrm{dex})$ and the turbulence velocity $\left(\mathrm{v}_{\mathrm{t}}=2 \mathrm{~km} \mathrm{~s}^{-1}\right)$. It is less than 0.08 dex. It is small compared to the error made in fitting the synthetic line profile to the observed spectra. The global error depends on the element (uncertainty in the position of the continuum) and was estimated to be between 0.20 and 0.25 dex by François et al. (2007). We adopted err $=0.2$ dex for $\mathrm{Sr}, \mathrm{Y}$, and $\mathrm{Zr}, 0.25$ dex for $\mathrm{Mo}, \mathrm{Ru}$, and $\mathrm{Pd}$, and 0.3 dex for $\mathrm{Ag}$.

\section{Analysis of the results}

Among the EMP and VMP stars, there is a very large scatter of neutron-capture element abundances. Some are europium-rich and other europium-poor. Since Eu can be formed almost only by the r-process, these stars are classified as r-rich or r-poor according to their $[\mathrm{Eu} / \mathrm{Fe}]$ value. It has been shown (but often overlooked) that, in the old stars, there is an excellent correlation between the two ratios $[\mathrm{Eu} / \mathrm{Fe}]$ and $[\mathrm{Ba} / \mathrm{Fe}]$. In Fig. $4 \mathrm{~A}$ $[\mathrm{Eu} / \mathrm{Ba}]$ is plotted vs $[\mathrm{Ba} / \mathrm{Fe}]$ for the stars studied from the LP First Stars and the stars studied in a similar way by Siqueira Mello et al. (2014) and Siqueira-Mello et al. (2015). In these very old stars, we found $[\mathrm{Eu} / \mathrm{Ba}]=0.51 \pm 0.09$ dex. The constancy of the ratio $[\mathrm{Eu} / \mathrm{Ba}]$ has also been noted by Mashonkina et al. (2010). In their Fig. 12, at low metallicity, below $[\mathrm{Fe} / \mathrm{H}]=-2.5$, they found $\mathrm{A}(\mathrm{Ba})-\mathrm{A}(\mathrm{Eu}) \approx 1.1 \operatorname{dex}$ or $[\mathrm{Eu} / \mathrm{Ba}] \approx 0.56 \operatorname{dex}$ in good agreement with our mean value. This value is close to the pure r-process value $[\mathrm{Eu} / \mathrm{Ba}] \approx 0.7 \mathrm{dex}$ (Banerjee et al. 2017).

In such old stars, europium-rich stars are also Ba-rich (and conversely). As a consequence, at very low metallicity since, in particular in turn-off stars and most metal-poor giants, the $\mathrm{Eu}$ lines are extremely weak and a very high resolution and $\mathrm{S} / \mathrm{N}$ are required to measure these lines (see for example Siqueira Mello et al. 2012), it is possible to use Ba as a proxy of Eu. From Fig. 4A, it can be deduced that in normal VMP stars $[\mathrm{Eu} / \mathrm{Fe}]=[\mathrm{Ba} / \mathrm{Fe}]+0.51 \pm 0.09($ or $[\mathrm{Eu} / \mathrm{H}]=[\mathrm{Ba} / \mathrm{H}]+0.51)$. This is of course generally not valid for carbon-rich metal-poor stars enriched in neutron-capture elements by mass transfer by a more massive companion during its AGB phase.

In the next sections, we compare the relative abundances of the neutron-capture elements in the 11 LP First Stars with the abundances measured in two classical stars: that is, CS 31082-001, the well-known r-rich star (Hill et al. 2002; Siqueira Mello et al. 2013); and HD 122563 (Honda et al. 2007), the well-known r-poor star. 

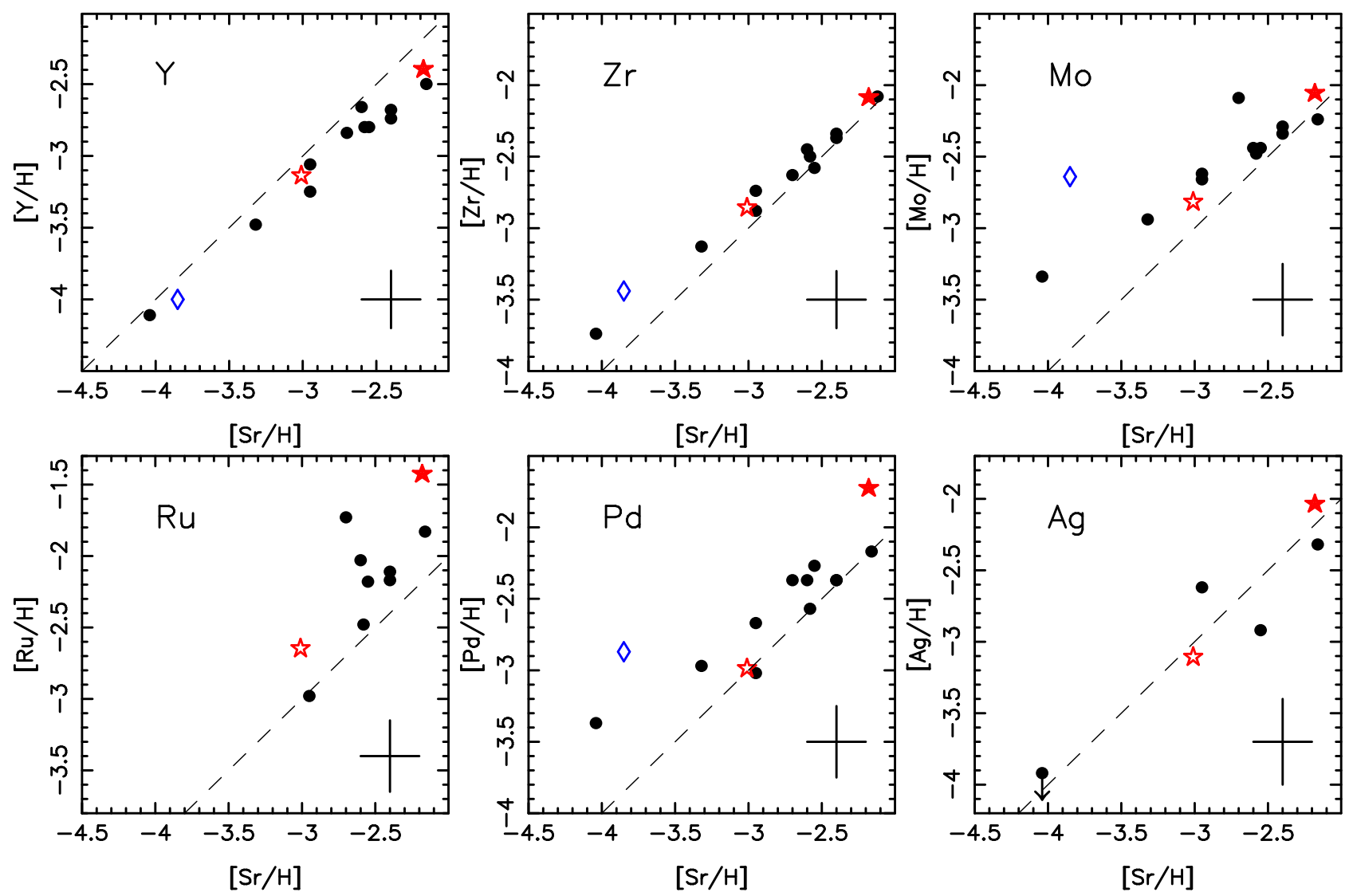

Fig. 2. Six relations between the ratios $[X / H]$ vs $[\mathrm{Sr} / \mathrm{H}]$ where $X$ is a first peak element, for the (up to) 11 "LP first stars" studied here (black dots). The blue open diamond represents the peculiar C-rich and Ba-poor star CS 22949-037. The two reference stars HD 122563 (r-poor star) and CS 31082-001 (r-rich star) are represented by an open and a filled red star symbol, respectively. The dashed line shows the one-to-one correspondence line. The typical error is indicated in the right corner of the figures.

Table 2. Metallicities $[\mathrm{Fe} / \mathrm{H}]$, and $\mathrm{A}(\mathrm{X})=\log \left(\mathrm{N}_{\mathrm{X}} / \mathrm{N}_{\mathrm{H}}\right)+12$ of the elements for the 7 neutron-capture elements of the first peak $(+\mathrm{A}(\mathrm{Ba})$ and $\mathrm{A}(\mathrm{Eu}))$ derived from LTE computations in our 11 "LP first stars".

\begin{tabular}{llrrrrrrrrrr}
\hline \hline ID & Object & {$[\mathrm{Fe} / \mathrm{H}]$} & $\mathrm{A}(\mathrm{Sr})$ & $\mathrm{A}(\mathrm{Y})$ & $\mathrm{A}(\mathrm{Zr})$ & $\mathrm{A}(\mathrm{Mo})$ & $\mathrm{A}(\mathrm{Ru})$ & $\mathrm{A}(\mathrm{Pd})$ & $\mathrm{A}(\mathrm{Ag})$ & $\mathrm{A}(\mathrm{Ba})$ & $\mathrm{A}(\mathrm{Eu})$ \\
& Sun* & 0.0 & 2.90 & 2.20 & 2.57 & 1.94 & 1.78 & 1.67 & 1.22 & 2.18 & 0.53 \\
& & & & & & & & & & & \\
1 & CS 22953-003 & -2.84 & 0.30 & -0.46 & 0.12 & -0.50 & -0.25 & -0.70 & - & -0.22 & -1.28 \\
2 & BD-18 5550 & -3.06 & -1.15 & -1.91 & -1.17 & -1.50 & $<-1.70$ & -1.70 & $<-2.70$ & -1.67 & -2.75 \\
3 & CS 22896-154 & -2.69 & 0.74 & -0.30 & 0.49 & -0.30 & -0.05 & -0.50 & -1.10 & -0.05 & -1.20 \\
4 & CS 22873-055 & -2.99 & -0.42 & -1.28 & -0.56 & -1.00 & $<-1.70$ & -1.30 & - & -1.31 & -2.65 \\
5 & BS 17569-049 & -2.88 & 0.35 & -0.60 & -0.01 & -0.50 & -0.40 & -0.60 & -1.70 & -0.55 & -1.65 \\
6 & HD 186478 & -2.59 & 0.50 & -0.54 & 0.22 & -0.35 & -0.37 & -0.70 & - & -0.42 & -1.60 \\
7 & CS 22966-057 & -2.62 & 0.20 & -0.64 & -0.06 & -0.15 & 0.05 & -0.70 & - & -0.73 & -1.80 \\
8 & HD 2796 & -2.47 & 0.50 & -0.48 & 0.21 & -0.40 & -0.35 & -0.70 & - & -0.48 & -1.77 \\
9 & CS 29518-051 & -2.69 & 0.41 & -0.51 & 0.16 & -0.50 & -0.70 & -0.90 & - & -1.01 & -2.01 \\
10 & CS 22873-166 & -2.97 & -0.05 & -0.86 & -0.17 & -0.70 & -1.20 & -1.30 & - & -1.54 & -2.76 \\
11 & CS 22897-008 & -3.41 & -0.05 & -1.05 & -0.31 & -0.70 & - & -1.00 & -1.40 & -2.36 & - \\
& & & & & & & & & & & \\
& CS 22949-037 & -3.97 & -0.95 & -1.80 & -0.87 & -0.70 & - & -1.20 & - & -2.52 & - \\
& CS 31082-001 & -2.90 & 0.72 & -0.19 & 0.49 & -0.11 & 0.36 & -0.05 & -0.81 & 0.40 & -0.72 \\
& HD 122563 & -2.77 & -0.11 & -0.93 & -0.28 & -0.87 & -0.86 & -1.31 & -1.88 & -1.65 & -2.77 \\
\hline
\end{tabular}

Notes. In column 1 lists the identification number ID of the star, also included in Fig. 4B. ID=1 is for the star with the lowest [Sr/Ba] ratio and $\mathrm{ID}=11$ for the star with the highest $[\mathrm{Sr} / \mathrm{Ba}]$ ratio (see $[\mathrm{Sr} / \mathrm{Ba}]$ in Table 3$)$. At the end of the table, we give the abundances of these elements in the peculiar C-rich star CS 22949-037 and in the classical r-rich and r-poor stars CS 31082-001 and HD 122563. (*)The solar abundances $A(X)_{\odot}$ are taken from Lodders et al. (2009). 
F. Spite et al.: Abundance patterns of the light neutron-capture elements in very and extremely metal-poor stars
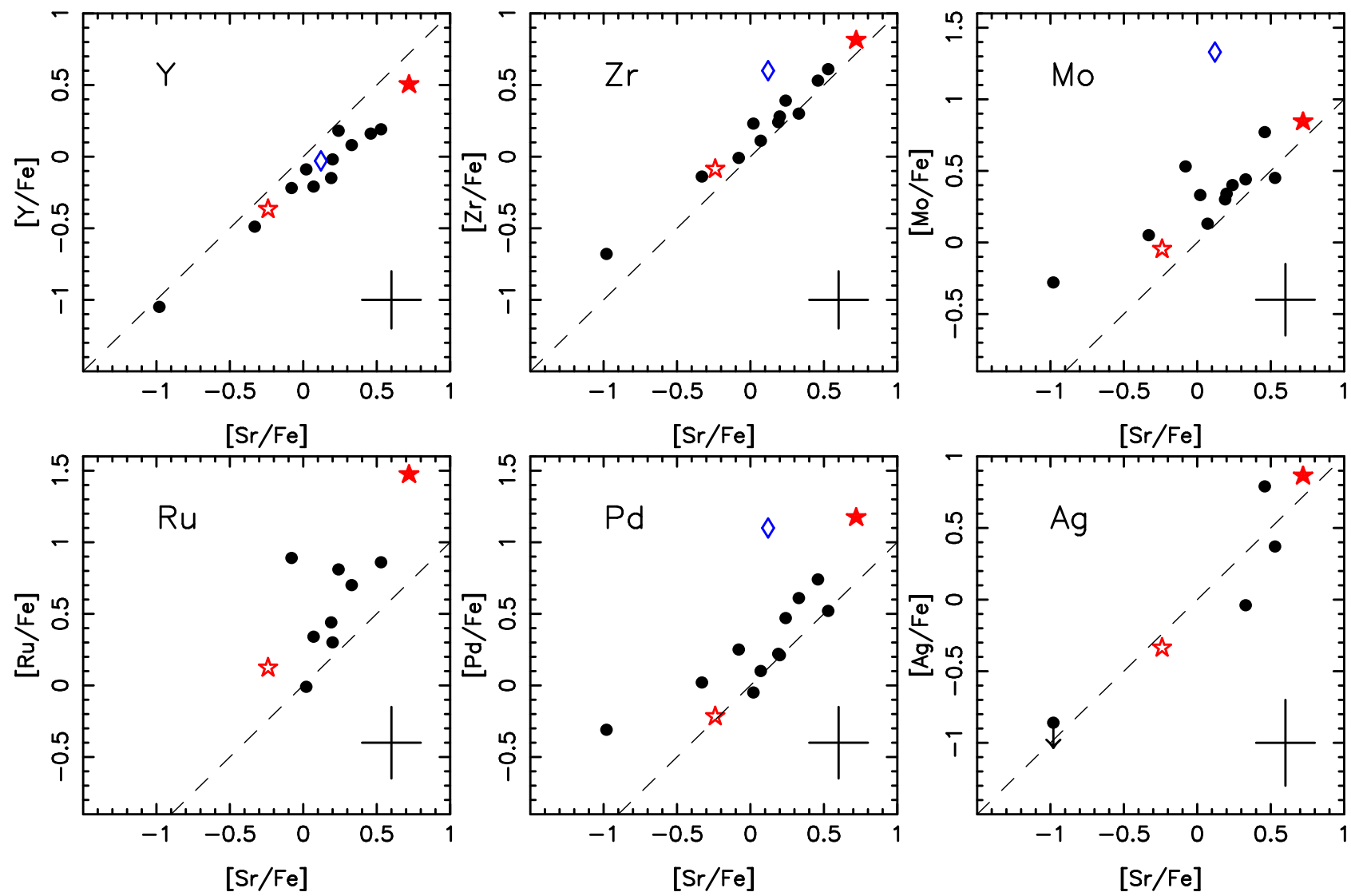

Fig. 3. Same as Fig. 2, but for $[\mathrm{X} / \mathrm{Fe}]$ vs $[\mathrm{Sr} / \mathrm{Fe}]$.
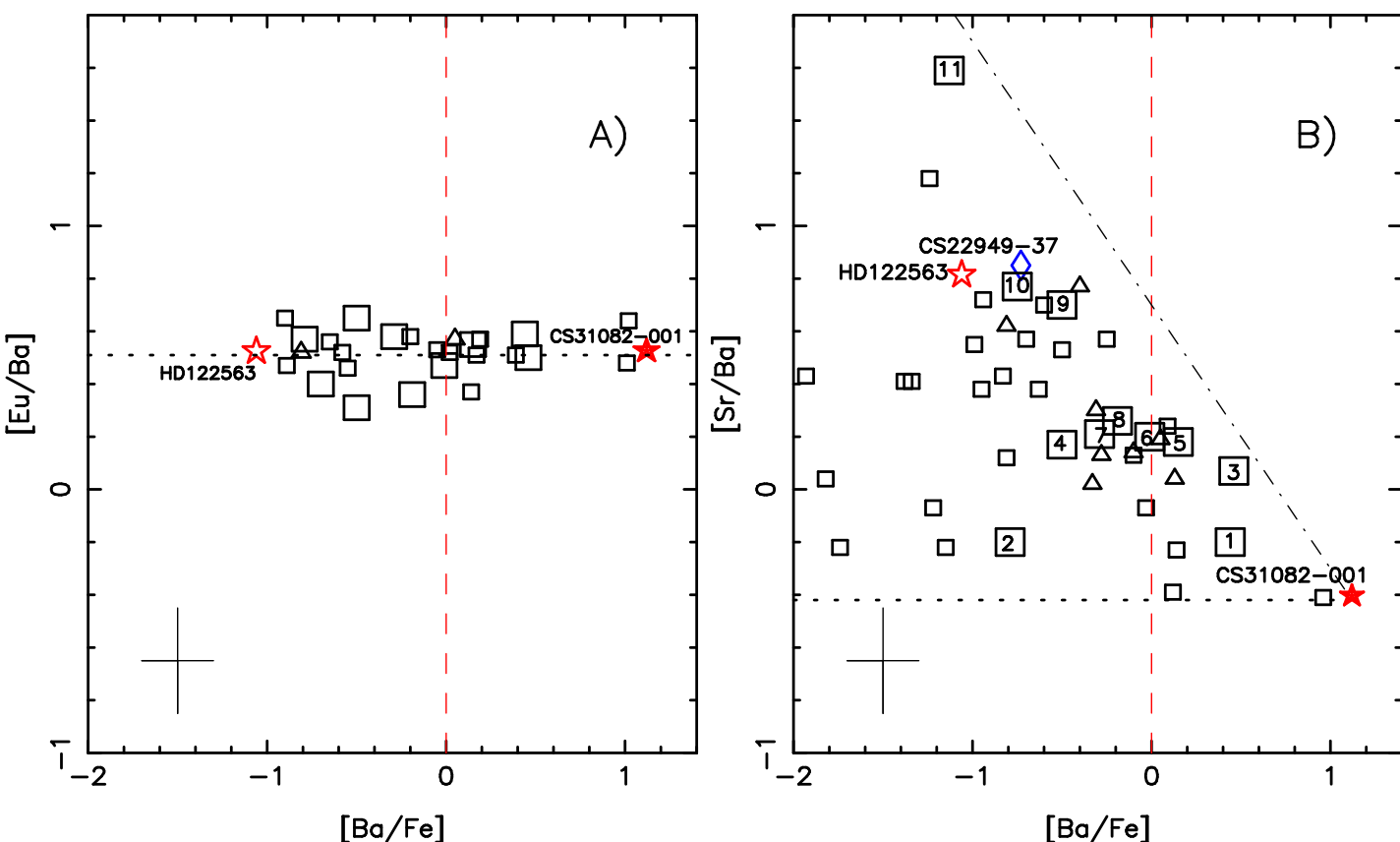

Fig. 4. $[\mathrm{Eu} / \mathrm{Ba}]$ and $[\mathrm{Sr} / \mathrm{Ba}]$ vs. $[\mathrm{Ba} / \mathrm{Fe}]$ for the stars studied from the LP First Stars and the stars studied by Siqueira Mello et al. (2014) and SiqueiraMello et al. (2015). The open triangles represent the turn-off stars and the open squares the giants. The big square symbols indicate the 11 giants studied here and the number given at the centre of the squares in panel $B$ refers to the identification of the star in Tables 2 and 3 (with higher numbers for higher [Sr/Ba]). CS 31082-001 (red filled star symbol) and HD 122563 (red open star symbol) are the =reference stars. CS 22949-037 (blue open diamond, only on panel $B$ ) is a peculiar C-rich metal-poor giant. In panels $A$ and $B$ a red dashed line is drawn at $[\mathrm{Ba} / \mathrm{Fe}]=0.0$; in panel $A$ the dotted line represents the mean LTE value of [Eu/Ba] in VMP stars (close to a pure $\mathrm{r}$ process value as observed in CS 31082-001) and in panel $B$ the minimum of $[\mathrm{Sr} / \mathrm{Ba}$ ] close to a pure r-process production as observed in CS 31082-001. The dash-dotted line in panel B indicates the upper limit of $[\mathrm{Sr} / \mathrm{Ba}]$ 
Table 3. Same as Table 2, but with $[\mathrm{X} / \mathrm{Fe}]$.

\begin{tabular}{|c|c|c|c|c|c|c|c|c|c|c|c|c|c|}
\hline ID & Object & {$[\mathrm{Fe} / \mathrm{H}]$} & {$[\mathrm{Sr} / \mathrm{Fe}]$} & [Y/Fe] & {$[\mathrm{Zr} / \mathrm{Fe}]$} & {$[\mathrm{Mo} / \mathrm{Fe}]$} & {$[\mathrm{Ru} / \mathrm{Fe}]$} & {$[\mathrm{Pd} / \mathrm{Fe}]$} & {$[\mathrm{Ag} / \mathrm{Fe}]$} & {$[\mathrm{Ba} / \mathrm{Fe}]$} & {$[\mathrm{Eu} / \mathrm{Fe}]$} & [Sr/Ba] & {$[\mathrm{Eu} / \mathrm{Ba}]$} \\
\hline 1 & CS 22953-003 & -2.84 & 0.24 & 0.18 & 0.39 & 0.40 & 0.81 & 0.47 & - & 0.44 & 1.03 & -0.20 & 0.59 \\
\hline 2 & BD-18 5550 & -3.06 & -0.99 & -1.05 & -0.68 & -0.38 & - & -0.31 & - & -0.79 & -0.22 & -0.20 & 0.57 \\
\hline 3 & CS 22896-154 & -2.69 & 0.53 & 0.19 & 0.61 & 0.45 & 0.86 & 0.52 & 0.37 & 0.46 & 0.96 & 0.07 & 0.50 \\
\hline 4 & CS 22873-055 & -2.99 & -0.33 & -0.49 & -0.14 & 0.05 & - & 0.02 & - & -0.50 & -0.19 & 0.17 & 0.31 \\
\hline 5 & BS 17569-049 & -2.88 & 0.33 & 0.08 & 0.30 & 0.44 & 0.70 & 0.61 & -0.04 & 0.15 & 0.70 & 0.18 & 0.55 \\
\hline 6 & HD 186478 & -2.59 & 0.19 & -0.15 & 0.24 & 0.30 & 0.44 & 0.22 & - & -0.01 & 0.46 & 0.20 & 0.47 \\
\hline 7 & CS 22966-057 & -2.62 & -0.08 & -0.22 & -0.01 & 0.53 & 0.89 & 0.25 & - & -0.29 & 0.29 & 0.21 & 0.58 \\
\hline 8 & HD 2796 & -2.47 & 0.07 & -0.21 & 0.11 & 0.13 & 0.34 & 0.10 & - & -0.19 & 0.17 & 0.26 & 0.36 \\
\hline 9 & CS 29518-051 & -2.69 & 0.20 & -0.02 & 0.28 & 0.25 & 0.21 & 0.12 & - & -0.50 & 0.15 & 0.70 & 0.65 \\
\hline 10 & CS 22873-166 & -2.97 & 0.02 & -0.09 & 0.23 & 0.33 & -0.01 & 0.00 & - & -0.75 & -0.32 & 0.77 & 0.43 \\
\hline 11 & CS 22897-008 & -3.41 & 0.46 & 0.16 & 0.53 & 0.77 & - & 0.74 & 0.79 & -1.13 & - & 1.59 & - \\
\hline & CS 22949-037 & -3.97 & 0.12 & -0.03 & 0.53 & 1.33 & - & 1.10 & - & -0.73 & - & 0.85 & - \\
\hline & CS 31082-001 & -2.90 & 0.72 & 0.51 & 0.82 & 0.8 & 1.48 & 1.18 & 0.87 & 1.12 & 1.65 & -0.43 & 0.53 \\
\hline & HD 122563 & -2.77 & -0.24 & -0.36 & -0.08 & -0.04 & 0.13 & -0.21 & -0.33 & -1.06 & -0.53 & 0.82 & 0.53 \\
\hline
\end{tabular}

Notes. $[\mathrm{Sr} / \mathrm{Ba}]$ and $[\mathrm{Eu} / \mathrm{Ba}]$ are given in columns 13 and 14 . The computations are carried out for $\mathrm{A}(\mathrm{Fe})_{\odot}=7.50$.
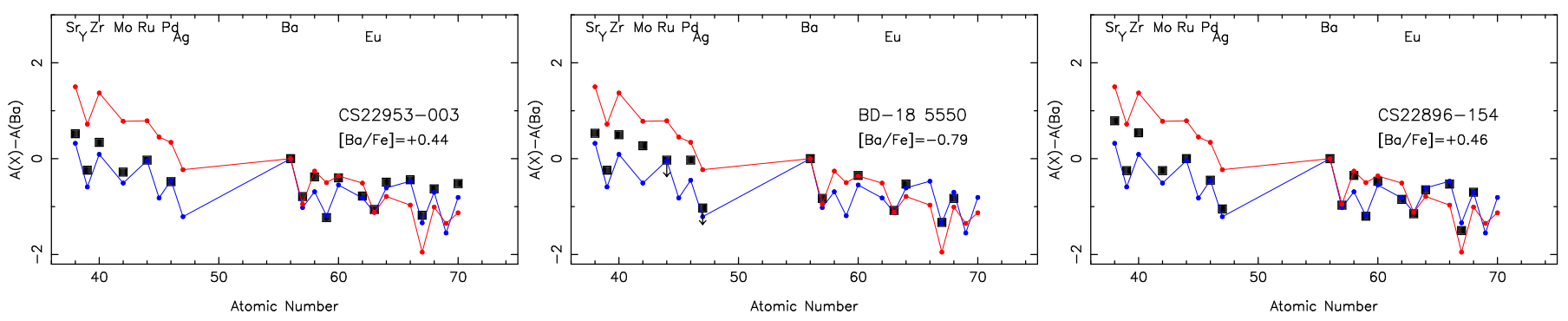

Fig. 5. Abundance patterns of the neutron capture elements for the stars of our sample with $[\mathrm{Sr} / \mathrm{Ba}]<0.10$. The blue line represents the pattern in CS 31082-001, which is a standard r-rich star, and the red line represents the pattern in HD 122563, which is an r-poor star with a high value of [Sr/Ba].

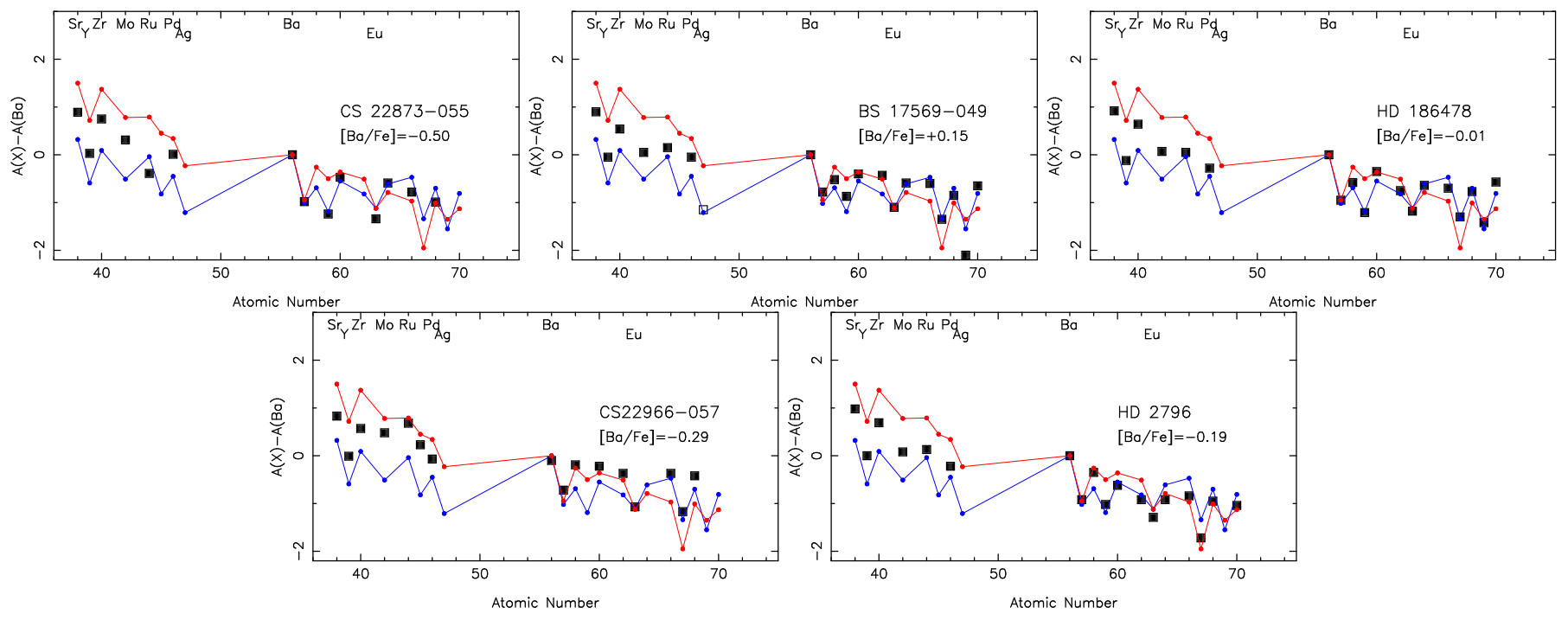

Fig. 6. Abundance patterns of the neutron capture elements for the stars of our sample with the intermediate ratios: $0.10<[\mathrm{Sr} / \mathrm{Ba}]<0.50$. The symbols are the same as in Fig. 5. 

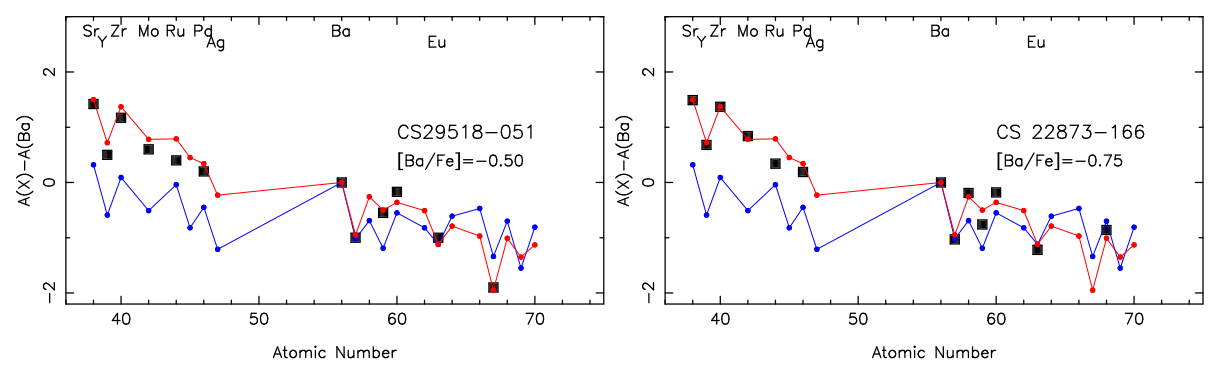

Fig. 7. Abundance patterns of the neutron capture elements for the two stars of our sample very similar to HD 122563 (with [Sr/Ba] 0.75). The symbols are the same as in Fig. 5.

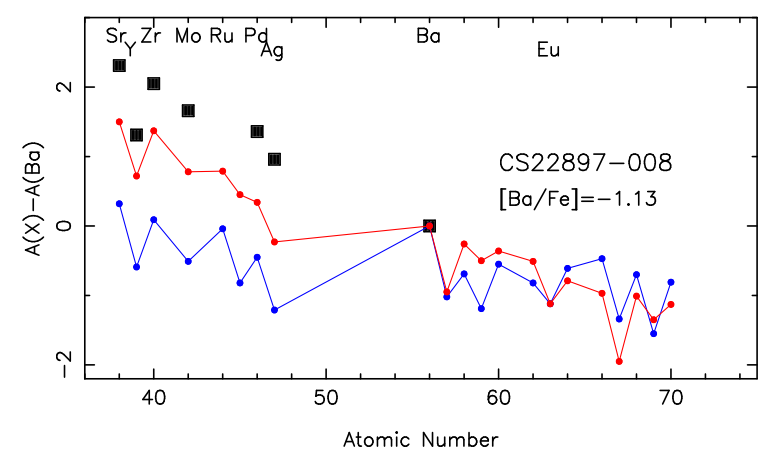

Fig. 8. Abundance pattern of the neutron capture elements in CS 22897008 (with $[\mathrm{Sr} / \mathrm{Ba}] \sim 1.6$ ), compared to CS 31082-001 (blue line) and HD 122563 (red line).

\subsection{Correlations between the abundances of the first peak elements}

Generally speaking, in all the EMP and VMP stars there is a rather good correlation between the abundance patterns of the second peak elements, extended from $\mathrm{Ba}$ to $\mathrm{Yb}$, (see e.g. François et al. 2007; Montes et al. 2007; Siqueira Mello et al. 2014). We wonder here about the abundances of the first peak (from $\mathrm{Sr}$ to $\mathrm{Ag}$ ).

In Fig. 2 we plot $[\mathrm{X} / \mathrm{H}]$ vs $[\mathrm{Sr} / \mathrm{H}]$, where $\mathrm{X}$ is for $\mathrm{Y}, \mathrm{Zr}$, Mo, $\mathrm{Ru}, \mathrm{Pd}$, and $\mathrm{Ag}$, and in Fig. $3[\mathrm{X} / \mathrm{Fe}]$ vs $[\mathrm{Sr} / \mathrm{Fe}]$. The excellent correlation between $[\mathrm{Y} / \mathrm{Fe}]$ and $[\mathrm{Sr} / \mathrm{Fe}]$ was already underlined by François et al. (2007). For the other elements of the first peak, the correlation is also good but the scatter is larger (see also Hansen et al. 2012).

\subsection{A peculiar object: CS 22949-37}

The star CS 22949-37 (Depagne et al. 2002; Norris et al. 2002) is classified as CEMP-no by Masseron et al. (2010), i.e. C-rich and EMP, without enhancement of the neutron-capture elements. The abundance anomalies in C-rich stars are often explained by recent pollution by a companion in its AGB phase. But the radial velocity $V_{R}$ of CS 22949-37 does not seem to be variable; in 2000 and 2001 Depagne et al. (2002) found $V_{R}=-125.64$ and $-125.62 \pm 0.2 \mathrm{~km} \mathrm{~s}^{-1}$ and in 2013 Starkenburg et al. (2014) measured $\mathrm{V}_{\mathrm{R}}=-125.9 \pm 0.3 \mathrm{~km} \mathrm{~s}^{-1}$. Therefore it is improbable that the star be a binary unless it is seen pole on. The abundances of this star should represent the abundances in the cloud that formed the star. Choplin et al. (2017) have explained the observed distribution of the elements (from $\mathrm{C}$ to $\mathrm{Fe}$ ) in single CEMP stars by the elements produced by fast rotating massive stars (FRMS). With its high overabundance of $\mathrm{Na}$ and $\mathrm{Mg}$ and with a ratio ${ }^{12} \mathrm{C} /{ }^{13} \mathrm{C}=4.0$, the element pattern of $\mathrm{CS} 22949-37$, is rather similar to the pattern of HE 2139-5432 fairly well represented by the model 20s7mix (Fig. 11 of Choplin et al. 2017).

In Fig. 3, the star CS 22949-37 has a normal [Y/Fe] ratio compared to the other LP first stars but it seems to be rich in $\mathrm{Zr}$, Mo, and Pd. The very weak lines of Ru could not be measured in this EMP star with $[\mathrm{Fe} / \mathrm{H}]=-4$.

\subsection{Sr/Ba scatter in metal-poor stars}

The abundance of the elements inside a given peak (first or second) are well correlated (see Fig. 3). But there is no clear correlation between the abundance of a first peak element (like $\mathrm{Sr}$ ) and the abundance of a second peak element (like Ba). For example in Fig. $4 \mathrm{~B}$ we plot the $[\mathrm{Sr} / \mathrm{Ba}]$ ratio vs $[\mathrm{Ba} / \mathrm{Fe}]$ for all the stars of the ESO LP First Stars and the stars studied in a homogeneous way by (Siqueira Mello et al. 2012; Siqueira-Mello et al. 2015). The position of the 11 EMP stars analysed here, are indicated, by numbered open squares. These stars are identified by numbers from 1 (for the star with the lowest $[\mathrm{Sr} / \mathrm{Ba}]$ ratio) to 11 for the star with the highest ratio (see Table 2). The position of the CEMP-no star CS 22949-37 is indicated with a blue diamond. All the stars in this figure have a metallicity $[\mathrm{Fe} / \mathrm{H}]$ below or equal to -2.5 , of which most are concentrated in the rather narrow range $[\mathrm{Fe} / \mathrm{H}]=-3.1 \pm 0.3$ dex.

Roederer (2013) remarked that in field stars the scatter of the relation $[\mathrm{Ba} / \mathrm{H}]$ vs $[\mathrm{Sr} / \mathrm{H}]$ increases when $[\mathrm{Ba} / \mathrm{H}]$ decreases. It is clear from Fig. 4B that the scatter of $[\mathrm{Sr} / \mathrm{Ba}]$ strongly increases when $[\mathrm{Ba} / \mathrm{Fe}]$ decreases (Spite \& Spite 2014; Spite et al. 2014; Jacobson et al. 2015). The horizontal dotted line in Fig. 4B represents the minimum of $[\mathrm{Sr} / \mathrm{Ba}]$ corresponding to its value in the r-rich stars and characteristic of the main r-process. The dashdotted line which, for each value of $[\mathrm{Ba} / \mathrm{Fe}]$, seems to mark the upper limit of $[\mathrm{Sr} / \mathrm{Ba}]$, corresponds to the line at $[\mathrm{Sr} / \mathrm{Ba}]=-$ $[\mathrm{Ba} / \mathrm{Fe}]+0.7$. The existence of this limit means in fact, that $[\mathrm{Sr} / \mathrm{Fe}]$ is never higher than about $+0.7 \mathrm{dex}$ a value close to the value observed (at this metallicity) in the r-rich stars, such as CS 31082-001, CS 22892-052 (Sneden et al. 2003; Sneden et al. 2008), CS 29497-004 (Hill et al. 2016), or RAVE J203843.2002333 (Placco et al. 2017). This is also the observed upper limit of $[\mathrm{Sr} / \mathrm{Fe}]$ at all metallicities following François et al. (2007, see their Fig. 1).

The stars with a ratio $[\mathrm{Ba} / \mathrm{Fe}]$ around -1 . dex, show a range of [Sr/Ba] spanning about 2 dex. For example, the star BD-18 5550 (labelled 2 in Fig. 4B) has about the same $\mathrm{Sr} / \mathrm{Ba}$ ratio as the classical r-rich star (CS 31082-001), but one of our stars (CS 22897-008, labelled 11 in Fig. 4B) has a $\mathrm{Sr} / \mathrm{Ba}$ ratio that is even higher than the ratio measured in the extreme star HD 122563.

Below $[\mathrm{Ba} / \mathrm{Fe}]=-1$, the scatter of $[\mathrm{Sr} / \mathrm{Ba}]$ does not continue to increase (it even seems to decrease) but the number of stars is very small and this partial trend is probably not significant. 


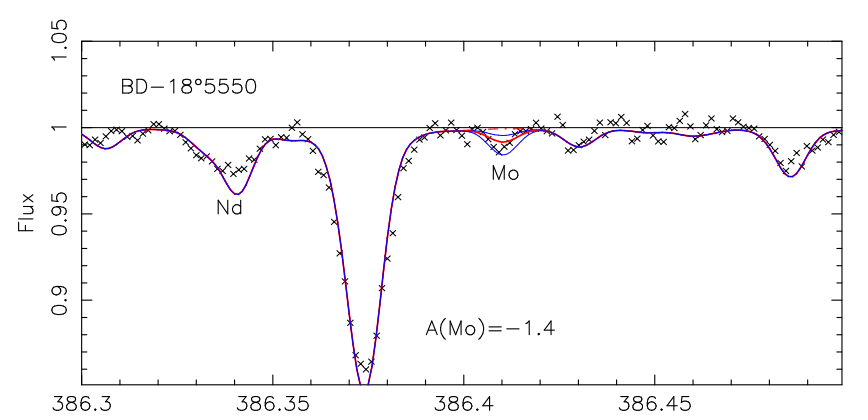

Fig. 9. Line of Mo in BD-185550. Crosses represent the observations. The synthetic profiles were computed with an absence of Mo (red dash-dotted line, here practically at the position of the continuum), the adopted abundance $\mathrm{A}(\mathrm{Mo})=-1.4$ (red thick line), and changing this abundance by \pm 0.3 dex (blue thin lines for $\mathrm{A}(\mathrm{Mo})=-1.7$ and -1.1 ).

\subsection{Abundance patterns of the neutron-capture elements in our sample of stars}

In Figs. 5-8 we present the abundance patterns of the neutroncapture elements in our sample of stars. The figures are sorted by increasing $[\mathrm{Sr} / \mathrm{Ba}]$. The abundances of the first peak elements are from Table 2, and the abundances of the second peak elements are from François et al. (2007) rescaled to the solar abundances of Lodders et al. (2009). The data of the first and second peak of the reference stars were taken from Siqueira Mello et al. (2013) for CS 31082-001 (blue line in the figures) and from Honda et al. (2007) for HD 122563 (red line). We did not find the Holmium $(\mathrm{Z}=67)$ abundance in HD 122563 in the literature; therefore, to allow a comparison with the stars here studied (Figs. 5 to 8), we measured the $345.6 \mathrm{~nm}$ line of Ho in the ESO spectra of this star and found $\mathrm{A}(\mathrm{Ho})=-3.6$, i.e. $[\mathrm{Ho} / \mathrm{Fe}]=-1.32$ (atomic data from Lawler et al. 2004).

\subsubsection{Stars with a $[\mathrm{Sr} / \mathrm{Ba}]$ ratio close to the low ratio observed in r-rich stars}

In this class we studied three stars with $[\mathrm{Sr} / \mathrm{Ba}]<+0.1$ (Fig. 5). Two of these stars, CS 22953-003 and CS 22896-154, for which $[\mathrm{Ba} / \mathrm{Fe}]=+0.44$ and $+0.46 \mathrm{dex}$, can be considered as r-rich but the third star, BD-18 5550, for which $[\mathrm{Ba} / \mathrm{Fe}]=-0.79$ (and $[\mathrm{Eu} / \mathrm{Fe}]=-0.22 \mathrm{dex})$, is an $\mathrm{r}$-poor star. In these three stars, the abundance pattern of the first peak elements is rather close to the pattern of the typical r-rich star CS 31082-001. In a recent paper, Roederer (2017) found also that the pattern of the n-capture elements in BD-185550 is close to the pattern of the main r-process. However we note that, in BD-18 5550, the abundance of molybdenum seems to be stronger than expected. In the region of the molybdenum line, the $\mathrm{S} / \mathrm{N}$ of the spectrum is close to 350 (Fig. 9) and we derived $\mathrm{A}(\mathrm{Mo})=-1.4 \pm 0.2$. This corresponds to 0.04 atoms of molybdenum for $10^{12} \mathrm{H}$ atoms, which is four times more than the predictions of a pure r-process. At this very low abundance of the neutron capture elements $([\mathrm{Ba} / \mathrm{H}] \approx-3.8)$ a very weak pollution is able to alter the abundance ratios.

Like BD-18 5550, three other stars studied in the LP First Stars associate a low $[\mathrm{Ba} / \mathrm{Fe}]$ ratio (below -1 ) and a low $[\mathrm{Sr} / \mathrm{Ba}]$ ratio (François et al. 2007). These stars are CS 22172-002, CS 29502-042, and CS 22885-096. We could not study the pattern of the first peak abundances in these stars because the $\mathrm{S} / \mathrm{N}$ of the obtained spectra did not allow the measurements of extremely weak lines.

\subsubsection{Stars with an intermediate $[\mathrm{Sr} / \mathrm{Ba}]$ ratio}

The five stars (Fig. 6) of our sample with a value of [Sr/Ba] higher than in CS 31082-001, but lower than in HD 122563, have a pattern of the neutron-capture elements intermediate between these two stars. This enhancement seems to be almost uniform for all the first peak elements that we measured. However in CS 22966-057 molybdenum seems to be more enhanced than the other first peak elements.

\subsubsection{Stars with a very high $[\mathrm{Sr} / \mathrm{Ba}]$ ratio}

In Figs. 7 and 8 the stars have a $[\mathrm{Sr} / \mathrm{Ba}]$ ratio higher than 0.7 dex. All these stars are r-poor with a low ratio $[\mathrm{Ba} / \mathrm{Fe}] \leq-0.5$. The two stars CS 29518-051 and CS 22873-166 (Fig. 7) have the same distribution of the neutron-capture elements as HD 122563; their first peak elements are enhanced by a factor of about ten relative to the classical r-rich star CS 31082-001.

One of the stars analysed here, CS 22897-008 (Fig. 8), seems to be even more extreme than HD 122563; compared to the classical r-rich star CS 31082-001, the first peak elements are almost uniformly enhanced by a factor of 100 . This extreme star has a very low $[\mathrm{Ba} / \mathrm{H}]$ ratio of -4.6 . The lines of the heaviest elements are not visible, and the estimated values of the corresponding equivalent widths are of the order of, or less than $1 \mathrm{~m} \AA$, which is obviously too small to be measured on our spectra.

\section{Discussion}

\subsection{Heavy elements enrichment in VMP and EMP stars}

Roederer (2013) noted that the yields of the heavy elements have to be decoupled from the production of magnesium and iron in order to explain the small observed dispersion in the ratios of light metals (such as $[\mathrm{Mg} / \mathrm{Fe}]$ ) and the large dispersion observed for $[\mathrm{Eu} / \mathrm{Fe}],[\mathrm{Ba} / \mathrm{Fe}]$, and $[\mathrm{Sr} / \mathrm{Fe}]$. In the previous sections, we have shown that in our sample of metal-poor stars with $[\mathrm{Fe} / \mathrm{H}]$ close to -3.0

- there is a good correlation among abundances of the neutron-capture elements belonging to the first peak (Figs. 2 and 3);

- the scatter of $[\mathrm{Sr} / \mathrm{Ba}]$ increases when $[\mathrm{Ba} / \mathrm{Fe}]$ decreases (Fig. 4B) whereas [Eu/Ba] remains constant (Fig. 4A); and

- many metal-poor stars (8 out of 11) have an abundance pattern of heavy elements that is clearly different from the main r-process predictions as represented by the abundance pattern in CS 31082-001 (Figs. 6-8).

Since at this low metallicity a contamination by classical sprocess yields from typical AGB stars was thought to be very improbable, the abundance pattern of HD 122563 was first explained by a peculiar process producing all the elements of the first and second peak: weak r-process in Honda et al. (2007), truncated r-process in Boyd et al. (2012), and also s-process in rotating massive VMP stars (Frischknecht et al. 2016) (see also Limongi \& Chieffi $2017^{2}$ ).

The fact that the spread in $[\mathrm{Sr} / \mathrm{Ba}]$ increases when $[\mathrm{Ba} / \mathrm{Fe}]$ decreases (Fig. 4B) rather suggests an enrichment in heavy elements of the matter in two independent steps. A first enrichment in pure r-process elements (as seen in CS 3102-001 and in the stars in Fig. 5) and, independently, an enrichment of mainly the first peak elements (as also suggested by Cescutti et al. 2013; Roederer 2017). This contamination by a second mechanism

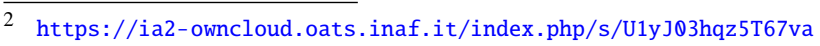




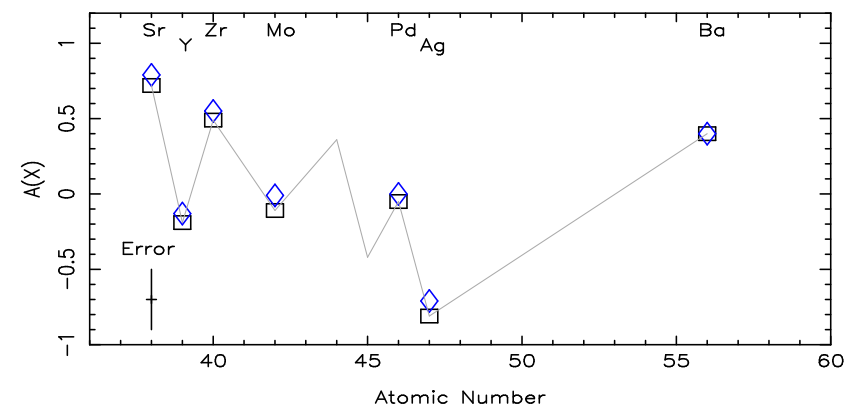

Fig. 10. Simulation of the effect of a pollution on the r-rich star CS 31082-001. We assume that a star such as CS31082-001 undergoes a pollution similar in atom numbers per $10^{12}$ hydrogen atoms as the pollution undergone by CS 22897-008. The black squares represent the abundances before pollution and the diamonds indicate the abundances after pollution. In such a star, the effect of the pollution is almost not visible.

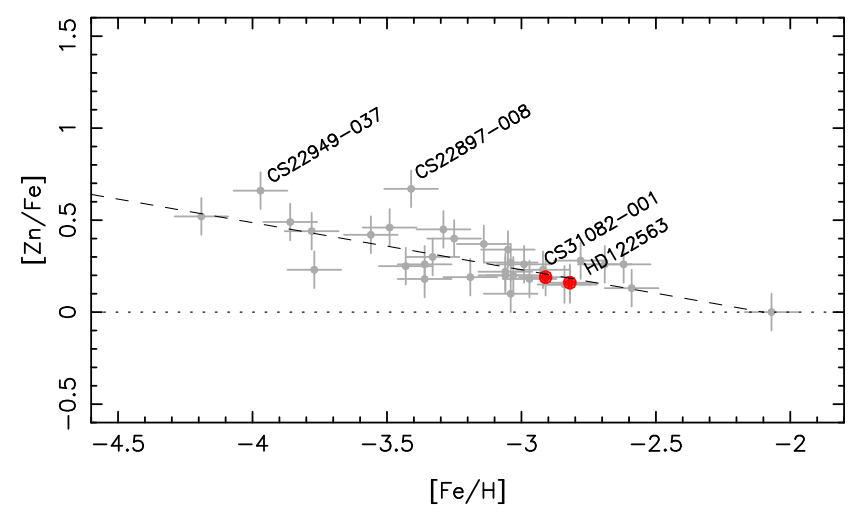

Fig. 11. Zn abundance in the VMP and EMP giants here studied. The position of the two reference stars CS 31082-001 (r-rich) and HD 122563 (r-poor but Sr-rich) stars are indicated by red filled circles and both have a normal position for their metallicity. On the contrary, CS 22897-008 and the CEMP star CS 22949-037 seem to be Zn-rich.

becomes visible and even dominates in stars that are originally r-poor such as HD 122563 and also in particular CS 22897-008 (Fig. 7). If an r-rich star undergoes this same contamination, the observed distribution of the neutron-capture elements abundances (and in particular the ratio $[\mathrm{Sr} / \mathrm{Ba}]$ ) is not significantly affected. We assume (Fig. 10) that CS 31082-001 undergoes a pollution similar (in number of atoms per $10^{12}$ atoms of hydrogen) to the pollution needed to explain in this context, the pattern of CS 22897-008. The star CS 31082-001 is already so rich in ncapture elements that the change in the observed distribution of the neutron-capture abundances is not significant. As a consequence, this second mechanism could be very frequent in the history of the metal enrichment of the Galaxy. An r-poor star with $[\mathrm{Sr} / \mathrm{Ba}]$ close to the pure r-process value (like BD-18 5550) would not have undergone a significant contamination by the second mechanism, unlike HD 122563 and the stars in Figs. 7 and 8.

In Fig. $4 \mathrm{~A}$, at a given value of $[\mathrm{Ba} / \mathrm{Fe}]$, the $[\mathrm{Sr} / \mathrm{Ba}]$ ratio depends on the importance of the contamination of the matter by the second mechanism. When a star is $r$ rich, a pollution similar to the pollution observed in some r-poor stars is not perceptible and the ratio $[\mathrm{Sr} / \mathrm{Ba}]$ remains constant. Thus at a given metallicity (here $[\mathrm{Fe} / \mathrm{H}]=-3.0$ ) the scatter of $[\mathrm{Sr} / \mathrm{Ba}]$ increases when $[\mathrm{Ba} / \mathrm{Fe}]$ decreases.

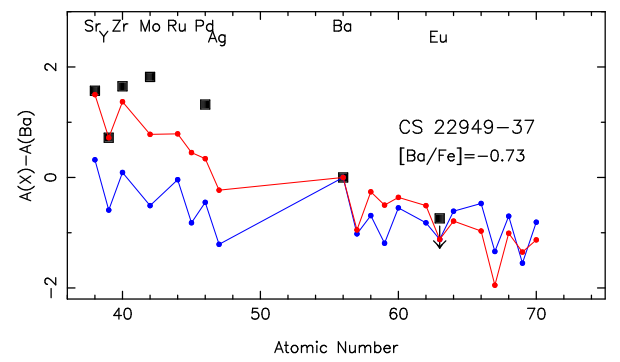

Fig. 12. Abundance pattern of the neutron capture elements in the CEMP star CS 22949-037 compared to CS 31082-001 (blue line) and HD 122563 (red line).

\subsection{CEMP star CS 22949-037}

In addition to the 11 classical VMP-EMP stars analysed here, we also considered the carbon-rich star CS 22949-037 (Tables 2, and 3), previously analysed in the context of the LP First Stars (Depagne et al. 2002). This star is EMP (with $[\mathrm{Fe} / \mathrm{H}]=-3.97$ ), $\mathrm{N}$-rich, and somewhat Zn-rich, but Ba-poor (Fig. 11). Since the abundance of $\mathrm{Zr}$ was not measured in Depagne et al. (2002), we measured the $\mathrm{Zr}$ lines in addition to Mo and Pd. In Fig. 3 this star stands out from the mean relations between the first peak elements observed in the EMP-VMP stars of our sample. Although the $\mathrm{Sr}$ and $\mathrm{Y}$ abundances seem to be normal, obviously, in this star, the abundances of $\mathrm{Zr}$, Mo, and Pd are enhanced.

The pattern of the element abundances (Fig. 12) is somewhat similar to the pattern of HD 122563 for Sr, Y, and Zr, but with higher abundances of Mo and Pd. This star is included in the paper by Frischknecht et al. (2016, their Sect. 6.2.2 and Table 8). These authors suggest (from only the few abundances available at this time, and mainly the $[\mathrm{Sr} / \mathrm{Ba}]$ ratio) that the peculiar pattern of CS 22949-037 could be explained by a FRMS model. It would be interesting to check whether in particular, $\mathrm{Zr}$, Mo, and Pd could be simultaneously explained.

\section{Conclusions}

The general distribution of the heavy elements in space and time, shows a very complex picture. It seems that it is possible to explain our detailed observations by an enrichment in n-capture elements in two different events (as proposed by Qian \& Wasserburg 2001, 2007; Hansen et al. 2014b). In one event the natal cloud would be enriched by pure r-process products of the primitive supernovae. In another event this material would be enriched in (mainly) the first peak elements. However, we stress that the data do not suggest in which order (if any) these two events occurred. We then explain the nature of the second process.

i) Following for example Wanajo (2013) various processes in supernovae (ECsupernovae) could overproduce the first peak elements (weak r-process), their production showing some variety. This variety is proposed by Aoki et al. (2017) to explain the abundance patterns of the five galactic stars they observed (in the range $-2.85<[\mathrm{Fe} / \mathrm{H}]<-2.1$ ) that are similar to the pattern of the intermediate stars in our Fig. 6.

ii) Also the production of first peak elements during the pulsations of AGB or SAGB (Bisterzo et al. 2014) stars could be invoked (Siqueira-Mello et al. 2016). This process could be important following Bisterzo et al. (2016, 2017).

iii) In a recent paper, Frischknecht et al. (2016) provided new data about FRMS stars, some of these were able to produce a high proportion of first peak elements. In this case, 
the large amount of neutrons, produced by the rapid rotation of the massive star, could transform the iron seed nuclei into heavy elements of mainly the first peak. The elements heavier than the first peak (and in particular $\mathrm{Ba}$ ) would not be produced (or only in very small amounts) by such a rotational process. This interesting theory has been recently confronted to the measurement of a star rich in first-peak neutron-capture elements (ROA 276) in the globular cluster $\Omega$ Cen. This peculiar star also presents an overabundance of $\mathrm{Cu}$ and $\mathrm{Zn}$ that is very well represented by the very specific pattern produced by the FRMS model. Unfortunately, in our VMP or EMP stars the abundance of $\mathrm{Cu}$ cannot be determined because the $\mathrm{Cu}$ lines in the visible, are too weak. But the abundance of $\mathrm{Zn}$ was measured in all the stars here studied (Cayrel et al. 2004). In HD 122563 (Fig. 11), the ratio $[\mathrm{Zn} / \mathrm{Fe}]$ is found to be normal, that is the same as the other stars of the same metallicity. But in this figure the more extreme star CS 22897-008 seems to be Zn-rich for its metallicity.

iv) One more possibility, following Nishimura et al. (2017), would be the "intermediate r-process in core-collapse supernovae driven by the magneto-rotational instability". This process also produces also an excess of $\mathrm{Zn}$ and could be also invoked to explain the peculiar pattern of CS 22897-008.

v) Banerjee et al. (2017) suggested that a significant number of protons could be ingested into the He shell at the end of the life of massive EMP stars (including zero-metal stars) of about 20 to $30 M_{\odot}$. This proton ingestion would induce a neutron flux that would react with primary $\mathrm{C}$ and $\mathrm{O}$ produced by He burning. Such nucleosynthesis would produce mainly $\mathrm{Sr}$ along with elements up to $\mathrm{Ba}$ (and beyond).

Acknowledgement. B.B. acknowledges partial financial support from Fapesp, $\mathrm{CNPq}$, and CAPES

\section{References}

Alvarez, R., Plez, B. 1998, A\&A 330, 1109

Aoki, M., Ishimaru, Y., Aoki, W., \& Wanajo, S. 2017, ApJ, 837, 8

Ballester, P., Modigliani, A., Boitquin, O., et al. 2000, The Messenger, 101, 31

Banerjee, P., Qian, Y.-Z., \& Heger, A. 2017, ArXiv e-print [arXiv: 1711.05964v1]

Bisterzo, S., Travaglio, C., Gallino, R., Wiescher, M., \& Käppeler, F. 2014, ApJ, 787,10

Bisterzo, S., Travaglio, C., Wiescher, M., et al. 2016, J. Phys. Conf. Ser., 665, 012023

Bisterzo, S., Travaglio, C., Wiescher, M., Käppeler, F., Gallino, R. 2017, ApJ, 835,97

Bonifacio, P., Spite, M., Cayrel, R., et al. 2009, A\&A, 501, 519

Boyd, R. N., Famiano, M. A., Meyer, B. S., et al. 2012, ApJ, 744, L14

Cayrel, R., Depagne, E., Spite, M., et al. 2004, A\&A, 416, 1117

Cescutti, G., \& Chiappini, C. 2014, A\&A, 565, A51

Cescutti, G., Chiappini, C., Hirschi, R., Meynet, G., \& Frischknecht, U. 2013, A\&A, 553, A51

Choplin, A., Ekström, S., Meynet, G., Maeder, A., Georgy, C., Hirschi, R. 2017, A\&A, 605, A63

Cowan, J. J., Roederer, I. U., Sneden, C., \& Lawler, J. E. 2011, RR Lyrae Stars, Metal-Poor Stars, and the Galaxy, 5, 223

Dekker, H., D'Odorico, S., Kaufer, A., Delabre, B., \& Kotzlowski, H. 2000, Proc. SPIE, 4008, 534

Depagne, E., Hill, V., Spite, M., et al. 2002, A\&A, 390, 187

François , P., Depagne, E., Hill, V., et al. 2007, A\&A, 476, 935
Freiburghaus, C., Rosswog, S., \& Thielemann, F.-K. 1999, ApJ, 525, L121 Frischknecht, U., Hirschi, R., Pignatari, M., et al. 2016, MNRAS, 456, 1803 Goriely S, Bauswein A, Janka H-T. 2015, ApJ, 738, L32

Grevesse, N., \& Sauval, A. J. 2000, Origin of Elements in the Solar System, Implications of Post-1957 Observations, 261

Gustafsson B., Bell R. A., Eriksson K., Nordlund Å., 1975, A\&A, 42, 407

Gustafsson B., Edvardsson B., Eriksson K., et al. 2003, in Stellar Atmosphere Modeling, eds. I. Hubeny, D. Mihalas, \& K. Werner, ASP Conf. Ser., 288, 331

Gustafsson B., Edvardsson B., Eriksson K., Graae-Jørgensen U., Nordlund Å., Plez B., 2008, A\&A, 486, 951

Hansen, C. J., \& Primas, F. 2011, A\&A, 525, L5

Hansen, C. J., Primas, F., Hartman, H., et al. 2012, A\&A, 545, A31

Hansen, C. J., Andersen, A. C., \& Christlieb, N. 2014a, A\&A, 568, A47

Hansen, C. J., Montes, F., \& Arcones, A. 2014b, ApJ, 797, 123

Hill, V., Plez, B., Cayrel, R., et al. 2002, A\&A, 387, 560

Hill, V., Christlieb, N., Beers, T. C., et al. 2017, A\&A, 607, A91

Hirschi, R., Maeder, A., Meynet, G., Chiappini, C., \& Ekström, S. 2007, EAS Pub. Ser., 24, 263

Honda, S., Aoki, W., Ishimaru, Y., Wanajo, S., \& Ryan, S. G. 2006, ApJ, 643, 1180

Honda, S., Aoki, W., Ishimaru, Y., \& Wanajo, S. 2007, ApJ, 666, 1189

Jacobson, H. R., Keller, S., Frebel, A., et al. 2015, ApJ, 807, 171

Käppeler F, Gallino R, Busso M, Picchio G, Raiteri CM. 1990, Rev. Mod. Phys., 83, 157

Kasen, D., Metzger, B., Barnes, J., Quataert, E., \& Ramirez-Ruiz, E. 2017 , Nature, 551, 80

Lawler, J.E., Sneden, C., Cowan, J.J., et al. 2004, ApJ, 604, 850

Limongi, M., \& Chieffi, A. 2017,

https://ia2-owncloud.oats.inaf.it/index.php/s/U1yJ03hqz5T67va

Lodders, K., Palme H., \& Gail, H.P. 2009, in Landolt-Bornstein, New Series, VI/4B, Chap. 4.4, ed. J.E. Truemper (Berlin, Heidelberg, New York: SpringerVerlag), 560

Mashonkina, L., Christlieb, N., Barklem, P. S., et al. 2010, A\&A, 516, A46

Masseron, T., Johnson, J. A., Plez, B., et al. 2010, A\&A, 509, A93

Meynet, G., Ekström, S., \& Maeder, A. 2006, A\&A, 447, 623

Montes, F., Beers, T. C., Cowan, J., et al. 2007, ApJ, 671, 1685

Nishimura, N., Sawai, H., Takiwaki, T., Yamada, S., \& Thielemann, F.-K. 2017, ApJ, 836, L21

Norris, J. E., Ryan, S. G., Beers, T. C., Aoki, W., \& Ando, H. 2002, ApJ, 569, L107

Peterson, R. C. 2011, ApJ, 742, 21

Peterson, R. C. 2013, ApJ, 768, L13

Placco, V. M., Holmbeck, E. M., Frebel, A., et al. 2017, ApJ, 844, 18

Plez, B., 2012, ascl.soft05004P, http://adsabs.harvard.edu/abs/ 2012ascl. soft05004P

Qian, Y.-Z., \& Wasserburg, G. J. 2001, ApJ, 549, 337

Qian, Y.-Z., \& Wasserburg, G. J. 2007, Phys. Rep., 442, 237

Roederer, I. U. 2013, AJ, 145, 26

Roederer, I. U. 2017, ApJ, 835, 23

Siqueira Mello, C., Barbuy, B., Spite, M., \& Spite, F. 2012, A\&A, 548, A42

Siqueira Mello, C., Spite, M., Barbuy, B., et al. 2013, A\&A, 550, A122

Siqueira Mello, C., Hill, V., Barbuy, B., et al. 2014, A\&A, 565, A93

Siqueira-Mello, C., Andrievsky, S. M., Barbuy, B., et al. 2015, A\&A, 584, A86

Siqueira-Mello, C., Chiappini, C., Barbuy, B., et al. 2016, A\&A, 593, A79

Sneden, C., Cowan, J. J., Lawler, J. E., et al. 2003, ApJ, 591, 936

Sneden, C., Cowan, J. J., \& Gallino, R. 2008, ARA\&A, 46, 241

Spite, M., \& Spite, F. 2014, Astron. Nachr., 335, 65

Spite, M., Cayrel, R., Plez, B., et al. 2005, A\&A, 430, 655

Spite, M., Spite, F., Bonifacio, P., et al. 2014, A\&A, 571, A40

Starkenburg, E., Shetrone, M. D., McConnachie, A. W., \& Venn, K. A. 2014 MNRAS, 441, 1217

Vangioni, E., Goriely, S., Daigne, F., François, P., \& Belczynski, K. 2016, MNRAS, 455, 17

Wanajo, S. 2013, ApJ, 770, L22

Wanajo, S., Janka, H.-T., \& Müller, B. 2011, ApJ, 726, L15

Wanajo S, Sekiguchi Y, Nishimura N., et al. 2014, ApJ, 789, L39

Winteler, C., Käppeli, R., Perego, A., et al. 2012, ApJ, 750, L22

Yong, D., Norris, J. E., Da Costa, G. S., et al. 2017, ApJ, 837, 176 